\title{
Isogeometric Shape Optimization of Vibrating Membranes
}

\author{
Nguyen, Dang Manh; Evgrafov, Anton; Gersborg, Allan Roulund; Gravesen, Jens
}

Published in:

Computer Methods in Applied Mechanics and Engineering

Link to article, DOI:

10.1016/j.cma.2010.12.015

Publication date:

2011

Document Version

Peer reviewed version

Link back to DTU Orbit

Citation (APA):

Nguyen, D. M., Evgrafov, A., Gersborg, A. R., \& Gravesen, J. (2011). Isogeometric Shape Optimization of Vibrating Membranes. Computer Methods in Applied Mechanics and Engineering, 200(13-16), 1343-1353. https://doi.org/10.1016/j.cma.2010.12.015

\section{General rights}

Copyright and moral rights for the publications made accessible in the public portal are retained by the authors and/or other copyright owners and it is a condition of accessing publications that users recognise and abide by the legal requirements associated with these rights.

- Users may download and print one copy of any publication from the public portal for the purpose of private study or research.

- You may not further distribute the material or use it for any profit-making activity or commercial gain

- You may freely distribute the URL identifying the publication in the public portal 


\title{
Isogeometric shape optimization of vibrating membranes
}

\author{
Nguyen Dang Manh ${ }^{\mathrm{a}}$, Anton Evgrafov ${ }^{\mathrm{a}}$, Allan Roulund Gersborg ${ }^{\mathrm{b}}$, Jens Gravesen ${ }^{\mathrm{a}, *}$ \\ ${ }^{a}$ Department of Mathematics, Technical University of Denmark, Matematiktorvet, Building 303S, \\ DK-2800 Kgs. Lyngby, Denmark \\ ${ }^{b}$ Department of Mechanical Engineering, Technical University of Denmark, Nils Koppels Allé, \\ Building 404, DK-2800 Kgs. Lyngby, Denmark
}

\begin{abstract}
We consider a model problem of isogeometric shape optimization of vibrating membranes whose shapes are allowed to vary freely. The main obstacle we face is the need for robust and inexpensive extension of a B-spline parametrization from the boundary of a domain onto its interior, a task which has to be performed in every optimization iteration. We experiment with two numerical methods (one is based on the idea of constructing a quasi-conformal mapping, whereas the other is based on a spring-based mesh model) for carrying out this task, which turn out to work sufficiently well in the present situation. We perform a number of numerical experiments with our isogeometric shape optimization algorithm and present smooth, optimized membrane shapes. Our conclusion is that isogeometric analysis fits well with shape optimization.
\end{abstract}

Keywords: Isogeometric analysis, shape optimization, B-spline parametrization, vibrating membrane, eigenvalue.

\section{Introduction}

Shape optimization is a classical mathematical problem with a multitude of applications in engineering disciplines; see for example the monographs $[1,2]$ and references therein. From the theoretical perspective, the most interesting cases occur when the shapes under consideration are not restricted to be diffeomorphic to each other, that is, when changes in the topology are allowed. Such problems are often treated by parametrizing the shape indirectly, using for example the coefficients of the partial differential equation governing an engineering model under consideration (control in the coefficients, homogenization, or topology optimization approaches, see [3, 4]) or auxiliary surfaces such as in level-set methods, see [5]. All the mentioned methods gain their computational efficiency from the fact that they are based on fixed grids, which provides a tremendous advantage particularly in $3 \mathrm{D}$.

\footnotetext{
${ }^{*}$ Corresponding author. Tel.: +45 4525 3064; fax: +45 45881399.

Email addresses: D.M.Nguyen@mat.dtu.dk (Nguyen Dang Manh), A.Evgrafov@mat.dtu.dk (Anton Evgrafov), agersborg.hansen@gmail.com (Allan Roulund Gersborg), J.Gravesen@mat.dtu.dk (Jens Gravesen)
} 
Having industrial applications in mind, it would be convenient to integrate geometry optimization into CAD environments. For this to be possible one needs to utilize a direct CAD-like representations of the boundary. Such a representation should be maintained at every optimization iteration by shape optimization methods at the expense of needing to re-generate or to update frequently the volumetric mesh, which is needed for solving equations governing a given system. This expense imposes a computational penalty on the total performance of shape optimization methods.

One promising method of combining the efficiency of the computations on a fixed grid with the demand of a direct CAD-like parametrization of the boundary within the shape optimization framework is utilizing isogeometric analysis (IGA) for numerically solving the equations governing a given engineering system [6-13]. In this way one keeps all the computations on a fixed mesh on a parameter domain while gaining the advantage that optimized geometries can be easily processed in CAD systems for manufacturing $[6,14]$.

In the present paper we utilize isogeometric analysis-based shape optimization (IGSO) for designing vibrating membranes with prescribed eigenvalues. We treat vibrating membranes as as a model problem for more general spectral shape optimization problems of systems governed by elliptic operators $[15,16]$. It is also closely related to eigenfrequency optimization problems of vibrating plates with holes [17-19]. The problem of designing vibrating membranes is by no means a novel one: for example Hutchinson and Niordson [20] computed shapes of drums where the first few eigenvalues were prescribed. In particular, they considered the design problem of a harmonic drum, namely, a membrane whose first four eigenfrequencies form a ratio of 2:3:3:4. (The reason for the double eigenvalues is that it seems impossible to design a drum with frequencies 2:3:4 [15, 21, 22]). The idea in [20] was to use a conformal map from the circular domain to the domain occupied by the drum and to perform the eigenfrequency analysis on the former domain. Note that this idea is similar to IGA in the sense that a parametrization of the domain is utilized. Kane and Schoenauer [23] later attacked the problem by genetic algorithms, while in the present work we utilize gradient-based algorithms within the IGSO framework. We emphasize that we consider the problem as a model on which we can illustrate various re-parametrization strategies within IGSO context.

In the present work, the only generic requirement we place on a family of candidate feasible shapes in the shape optimization problems we consider is that they are diffeomorphic to each other. Whereas this requirement may be viewed as a restrictive one from the theoretical perspective, it is much more general than what is often considered within the shape optimization framework and leads to certain computational challenges. This is in a stark contrast with the situations when domain families parametrized by only a few variables are consedered (such as, for example, a circle of a varying radius [11, 24], or a family of super-elliptical shapes $[17,18]$ ), or when only certain parts of the boundary are allowed to vary locally $[10,13,24,25]$. Restrictions on the variations of the shape simplify significantly the task of remeshing in a FEM-based shape optimization, or the task of extending the parametrization from the boundary into the interior of the domain in IGSO-based shape optimization.

Owing to the richness of the family of shapes which we allow, constructing the expension of parametrization from the boundary into the interior of the domain becomes a non-trivial task in the present situation. We experiment with two linear methods for computing such an extension numerically: one is based on a spring model of the mesh and the other one is based on the idea of a quasi-conformal deformation. The former 
method is inspired by ideas coming from linear elasticity and works well for problems with convex domains. The strategy for the latter method is to find a well-behaving B-spline parametrization of an initial reference shape by solving auxiliary optimization problems, and then generate the inner control points by "quasi-conformally deforming" the reference shape into the resulting configuration. The procedure is repeated if an invalid parametrization appears at some shape optimization iteration.

The remainder of this paper is organized as follows. In Section 2, we briefly recall the equations governing vibrating membranes and their Galerkin discretization. The IGA model used in the present work and necessary techniques of handling a B-spline parametrization are presented in Section 3. In Section 4, we state the IGSO problem formulation and its sensitivity analysis. Our numerical experiments with IGSO are reported in Section 5. We conclude the paper with a summary of the results.

\section{Physical problem}

In this section we briefly recall the partial differential equations governing harmonic oscillations of a membrane and their Galerkin discretization.

\subsection{Governing equation}

Let $\Omega$ be a membrane whose circumference $\Gamma$ is constrained to be motionless. The out-of-plane displacement $U(\mathbf{x}, t)$ of a point $\mathbf{x} \in \Omega$ at time $t$ obeys the wave equation with homogeneous Dirichlet boundary condition

$$
\begin{aligned}
\frac{\partial^{2} U(\mathbf{x}, t)}{\partial t^{2}} & =c^{2} \Delta U(\mathbf{x}, t) & & \forall \mathbf{x} \in \Omega \\
U(\mathbf{x}, t) & =0 & & \forall \mathbf{x} \in \Gamma
\end{aligned}
$$

where $\Delta$ is the spatial Laplacian operator and $c$ is the wave speed, depending on the tension and the surface density of the membrane (c.f. [26]). Without losing generality, in what follows we assume that $c=1$.

The time-harmonic solutions to (1) having the form

$$
U(\mathbf{x}, t)=u(\mathbf{x}) e^{i \sqrt{\lambda} t},
$$

where $i^{2}=-1$ and $\lambda=(2 \pi f)^{2}$ with $f$ being the vibration frequency, are the pure tones the membrane is capable of producing (c.f. $[20,27]$ ).

Substituting (3) into (1) and (2) we recover Helmholtz equation with Dirichlet boundary condition

$$
\begin{aligned}
\Delta u+\lambda u & =0 & & \text { in } \Omega \\
u & =0 & & \text { on } \Gamma .
\end{aligned}
$$

The eigenfunctions $u$ are customarily normalized so that $\int_{\Omega} u^{2} \mathrm{~d} V=1$. 


\subsection{Weak form and discretization}

Let $H_{0}^{1}(\Omega)$ be the subspace of the Sobolev space $H^{1}(\Omega)$ (c.f. [28]) containing functions which vanish on the boundary. In its weak form, the homogeneous boundary value problem (4), (5) can be stated as follows: find $u \in H_{0}^{1}(\Omega)$ such that for every $v \in H_{0}^{1}(\Omega)$ it holds that

$$
\int_{\Omega} \nabla u \cdot \nabla v \mathrm{~d} V=\lambda \int_{\Omega} u v \mathrm{~d} V .
$$

Applying the Galerkin method (c.f. [29]) to (6) by approximating $H_{0}^{1}(\Omega)$ with conforming finite-dimensional subspaces to be described in Section 3.1 one arrives at the following generalized eigenvalue problem:

$$
\mathbf{K u}=\lambda \mathbf{M u}
$$

where $\mathbf{K}$ and $\mathbf{M}$, are the stiffness and the mass matrices, respectively. The eigenvectors in (7) are customarily normalized as

$$
\mathbf{u}^{T} \mathbf{M u}=1
$$

Later on, components of $\mathbf{u}$ will be referred to as the state variables to distinguish them from design variables.

\section{Isogeometric Analysis}

Isogeometric analysis (IGA) has recently been introduced by Hughes et al. [6] and has already found many applications in a variety of engineering disciplines [14]. The basic idea of IGA is to parametrize the domain, its boundary, and the solution space using B-splines defined by a single pair of knot vectors. For the purposes of isogeometric shape optimization this approach is modified, as described in the present section. We also discuss several approaches towards extending B-spline parametrizations from the boundary of the domain into its interior, validating the resulting parametrization, and improving its quality.

\subsection{Isogeometric analysis model}

Let us consider a simply connected domain $\Omega \subset \mathbb{R}^{2}$. In the present work, we use three different pairs of knot vectors for parametrizing the boundary $\Gamma=\partial \Omega$, the domain $\Omega$, and the solution space for (6), see Fig. 1. Our starting point is the pair of knot vectors $\boldsymbol{\Xi}_{u}$ and $\boldsymbol{\Xi}_{v}$ parametrizing the domain boundary with B-splines of degrees $p$ and $q$. Let $\boldsymbol{\rho}_{b}$ be the vector of the control point components of the boundary parametrization.

The second step is extending the boundary parametrization onto the interior of the domain $\Omega$. Here we may use a "finer" pair of knot vectors $\widehat{\boldsymbol{\Xi}}_{u}$ and $\widehat{\boldsymbol{\Xi}}_{v}$, obtained by inserting new knots into the knot vectors $\boldsymbol{\Xi}_{u}$ and $\boldsymbol{\Xi}_{v}$ respectively. The vector $\widehat{\boldsymbol{\rho}}_{b}$ containing components of the resulting boundary control points is obtained as

$$
\widehat{\boldsymbol{\rho}}_{b}=\mathbf{T} \boldsymbol{\rho}_{b}
$$

where $\mathbf{T}$ is a constant matrix depending only on the knot vectors [30]. Given the vector of the boundary control points $\widehat{\boldsymbol{\rho}}_{b}$ one may determine the inner control points $\widehat{\mathbf{d}}_{i, j}$, 


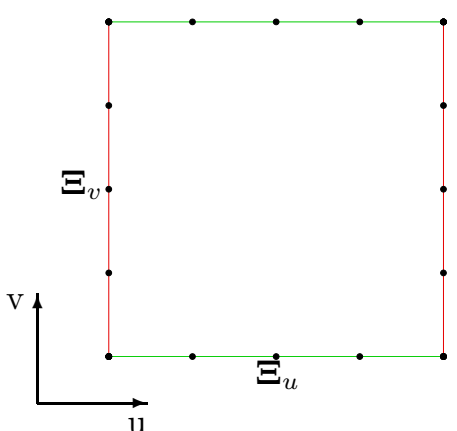

(a)

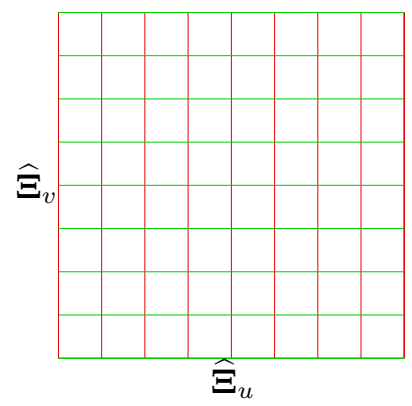

(b)

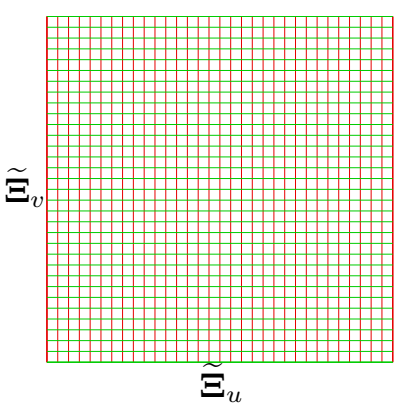

(c)

Figure 1: Three pairs of knot vectors of the IGA model used in the present work. (a): Knot vectors for the boundary parametrization; (b): knot vectors for the domain parametrization; (c): knot vectors for the solution space parametrization. The green and red lines correspond to horizontal and vertical parameter lines, respectively.

$i=1, \ldots, \widehat{n}, j=1, \ldots, \widehat{m}$ (the precise procedure will be discussed in Section 3.3). In turn, they parametrize the domain $\Omega=\left\{\mathbf{x} \in \mathbb{R}^{2} \mid \mathbf{x}=\mathbf{F}(u, v),(u, v) \in[0,1]^{2}\right\}$, where

$$
\mathbf{F}(u, v)=\sum_{i=1}^{\widehat{m}} \sum_{j=1}^{\widehat{n}} \widehat{\mathbf{d}}_{i, j} \widehat{M}_{i}^{p}(u) \widehat{N}_{j}^{q}(v) .
$$

In (10), $\widehat{M}_{i}^{p}$ and $\widehat{N}_{i}^{q}$ are B-splines of degree $p$ and $q$ with the knot vectors $\widehat{\boldsymbol{\Xi}}_{u}$ and $\widehat{\boldsymbol{\Xi}}_{v}$, respectively.

Suppose that the map $\mathbf{F}$ in $(10)$ is found to be a parametrization of $\Omega$, that is, the determinant of its Jacobian is positive (or negative) everywhere. Let $\mathbf{F}^{-1}: \Omega \rightarrow[0,1]^{2}$ be the inverse of $\mathbf{F}$. The solution space $\mathcal{S}$ approximating $H_{0}^{1}(\Omega)$ will be defined in terms of functions $\widetilde{R}_{k}^{\widetilde{p}, \widetilde{q}}:[0,1]^{2} \rightarrow \mathbb{R}, k=1, \ldots, \widetilde{m} \widetilde{n}$ and $\mathbf{F}^{-1}$ as $\mathcal{S}=\operatorname{span}\left\{\widetilde{R}_{k}^{\widetilde{p}, \widetilde{q}} \circ \mathbf{F}^{-1} \mid k=\right.$ $1, \ldots, \tilde{m} \tilde{n}\}$. To ensure that we can approximate any function in $H_{0}^{1}(\Omega)$ sufficiently well, we may want to use an even finer (when compared to $\widehat{\boldsymbol{\Xi}}_{u}$ and $\widehat{\boldsymbol{\Xi}}_{v}$ ) pair of knot vectors $\widetilde{\boldsymbol{\Xi}}_{u}$ and $\widetilde{\boldsymbol{\Xi}}_{v}$, see Fig. 1. Thus we define $\widetilde{M_{i}} \widetilde{p}, i=1, \ldots, \widetilde{m}$ and $\widetilde{N}_{j}^{\widetilde{q}}, j=1, \ldots, \widetilde{n}$ as the B-splines of degree $\widetilde{p}$ and $\widetilde{q}$ (not neccesary equal to $p$ and $q$ ) with the knot vector $\widetilde{\boldsymbol{\Xi}}_{u}$ and $\widetilde{\Xi}_{v}$, respectively. Finally, the splines $\widetilde{R}_{k}^{\widetilde{p}, \widetilde{q}}$ involved in the definition of $\mathcal{S}$ are defined as the tensor product splines $\widetilde{R}_{k}^{\widetilde{p}, \widetilde{q}}(u, v)=\widetilde{M}_{i}^{\widetilde{p}}(u) \widetilde{N}_{j}^{\widetilde{q}}(v), k=(\widetilde{n}-1) i+j$. Given the structure of $\mathcal{S}$, the entries of the stiffness and mass matrices in the discretized form (7) are computed as

$$
\begin{aligned}
K_{k \ell}=\iint_{[0,1]^{2}}\left(\mathbf{D} \widetilde{R_{k}^{p}, \widetilde{q}}(u, v) \mathbf{J}^{-1}\right)^{T} \mathbf{D} \widetilde{R}_{\ell}^{\widetilde{p}, \widetilde{q}}(u, v) \mathbf{J}^{-1} \operatorname{det}(\mathbf{J}) \mathrm{d} u \mathrm{~d} v, & k, \ell=1, \ldots, \widetilde{m} \tilde{n}, \\
M_{k \ell}=\iint_{[0,1]^{2}} \widetilde{R}_{k}^{\widetilde{p}, \widetilde{q}}(u, v) \widetilde{R}_{\ell}^{\widetilde{p}, \widetilde{q}}(u, v) \operatorname{det}(\mathbf{J}) \mathrm{d} u \mathrm{~d} v, & k, \ell=1, \ldots, \widetilde{m} \tilde{n},
\end{aligned}
$$

where $\mathbf{J}$ is the Jacobian of the parametrization $\mathbf{F}$ and $\mathbf{D} f(u, v)=\left[\frac{\partial f}{\partial u}(u, v) \quad \frac{\partial f}{\partial v}(u, v)\right]$ 
is the differential of a real-valued function $f:[0,1]^{2} \rightarrow \mathbb{R}$.

\subsection{Validating a B-spline parametrization}

In order to ensure that a given choice of inner control points $\widehat{\mathbf{d}}_{i, j}, i=1, \ldots, \widehat{n}$, $j=1, \ldots, \widehat{m}$ results in a valid B-spline parametrization of $\Omega$ we employ the following approach.

The determinant of a Jacobian of $\mathbf{F}$ given by (10) is computed as

$$
\operatorname{det}(\mathbf{J})=\sum_{i, j=1}^{\widehat{m}, \widehat{n}} \sum_{k, \ell=1}^{\widehat{m}, \widehat{n}} \operatorname{det}\left[\widehat{\mathbf{d}}_{i, j}, \widehat{\mathbf{d}}_{k, \ell}\right] \frac{\mathrm{d} \widehat{M}_{i}^{p}(u)}{\mathrm{d} u} \widehat{N}_{j}^{q}(v) \widehat{M}_{k}^{p}(u) \frac{\mathrm{d} \widehat{N}_{\ell}^{q}(v)}{\mathrm{d} v},
$$

where $\operatorname{det}\left[\widehat{\mathbf{d}}_{i, j}, \widehat{\mathbf{d}}_{k, \ell}\right]$ is the determinant of the $2 \times 2$ matrix with columns $\widehat{\mathbf{d}}_{i, j}, \widehat{\mathbf{d}}_{k, \ell}$. Equation (13) defines a piecewise polynomial of degree $2 p-1$ in $u$ and degree $2 q-1$ in $v$, which is $C^{p-2}$ in $u$ and $C^{q-2}$ in $v$. Such a map can be written in terms of B-splines $\mathcal{M}_{k}^{2 p-1}$ and $\mathcal{N}_{\ell}^{2 q-1}$ of degree $2 p-1$ and $2 q-1$ with the knot vectors obtained from $\widehat{\boldsymbol{\Xi}}_{u}$ and $\widehat{\boldsymbol{\Xi}}_{v}$ by raising the multiplicities of the inner $u$-knots and $v$-knots by $p$ and $q$, respectively [31]. That is

$$
\operatorname{det}(\mathbf{J})=\sum_{k, \ell=1}^{M, N} c_{k, \ell} \mathcal{M}_{k}^{2 p-1}(u) \mathcal{N}_{\ell}^{2 q-1}(v),
$$

where the coefficients $c_{k, \ell}$ depend linearly on the quantities $\operatorname{det}\left[\widehat{\mathbf{d}}_{i, j}, \widehat{\mathbf{d}}_{k, \ell}\right]$. Equation (14) tells us that if all $c_{k, \ell}$ are positive, then so is the determinant.

\subsection{Extending a B-spline parametrization from the boundary to the interior domain}

The only part needed to complete our IGA model of the vibrating membrane is the computation of interior control points $\widehat{\mathbf{d}}_{i, j}$ defining $\mathbf{F}$ from the vector of boundary control points $\widehat{\boldsymbol{\rho}}_{b}$. Since we have to perform this calculation during every shape optimization iteration, we are looking for computationally inexpensive methods, which would preferably result in parametrizations (in the sense of Section 3.2) of the domain. Two methods described in the following subsections perform relatively well in both categories, in our computational experience. Both methods are linear, that is, they can be written as

$$
\widehat{\boldsymbol{\rho}}=\mathbf{G} \widehat{\boldsymbol{\rho}}_{b},
$$

for some matrix $\mathbf{G}$, where $\widehat{\boldsymbol{\rho}}$ is the vector containing all components of all control points $\widehat{\mathbf{d}}_{i, j}$.

\subsubsection{The spring model}

Imagine that the edges of the control net are replaced by equally stiff linear elastic springs. Then the locations of the inner control boundary nodes $\widehat{\mathbf{d}}_{i, j}$ at the static force equilibrium, which are uniquely determined by the positions of the boundary control nodes, are the locations taken by this method. More precisely, $\widehat{\mathbf{d}}_{i, j}$ satisfy the equations

$$
\widehat{\mathbf{d}}_{i, j}=\left(\widehat{\mathbf{d}}_{i, j-1}+\widehat{\mathbf{d}}_{i+1, j}+\widehat{\mathbf{d}}_{i, j+1}+\widehat{\mathbf{d}}_{i-1, j}\right) / 4,
$$

where $\widehat{\mathbf{d}}_{i, j-1}, \widehat{\mathbf{d}}_{i+1, j}, \widehat{\mathbf{d}}_{i, j+1}$, and $\widehat{\mathbf{d}}_{i-1, j}$ are the neighbouring nodes attached to $\widehat{\mathbf{d}}_{i, j}$ with imaginary springs. Being diagonally dominant and irreducible, this system of linear equations admits a unique solution. 


\subsubsection{The quasi-conformal deformation}

Assume we have found a reference control net with desirable properties, in particular of being a parametrization of the domain. Let $\widehat{\mathbf{v}}$ and $\widehat{\mathbf{w}}$ be two adjacent edges of the control net as depicted in Fig. 2. The rotation matrix $\mathbf{R}(\widehat{\theta})$, with $\widehat{\theta}$ being the angle

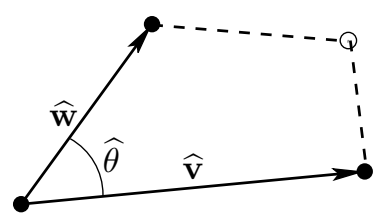

Figure 2: A local configuration in a reference control net

between $\widehat{\mathbf{v}}$ and $\widehat{\mathbf{w}}$, enjoys the identity

$$
\|\widehat{\mathbf{w}}\| \mathbf{R}(\widehat{\theta}) \widehat{\mathbf{v}}=\|\widehat{\mathbf{v}}\| \widehat{\mathbf{w}} .
$$

Consider now a new control net with $\mathbf{v}$ and $\mathbf{w}$ being the adjacent edges corresponding to $\widehat{\mathbf{v}}$ and $\widehat{\mathbf{w}}$ of the reference control net. For each such pair of edges we consider linear equations

$$
\|\widehat{\mathbf{w}}\| \mathbf{R}(\widehat{\theta}) \mathbf{v}=\|\widehat{\mathbf{v}}\| \mathbf{w} .
$$

The resulting linear system is overdetermined (there are $8(\widehat{m}-2)(\widehat{n}-2)$ equations with only $2(\widehat{m}-2)(\widehat{n}-2)$ unknown nodal positions) and is solved in the least squares sense.

\subsection{Improving a B-spline parametrization}

As none of the linear methods for extending the parametrization of the boundary into the interior of the domain can in general guarantee that the resulting map $\mathbf{F}$ will satisfy $\operatorname{det}(\mathbf{J})>0$ everywhere on $[0,1]^{2}$, sometimes we have to utilize a more expensive non-linear method for improving the distribution of the interior control points $\widehat{\mathbf{d}}_{i, j}$. One natural approach to ensure that $\operatorname{det}(\mathbf{J})$ is bounded away from zero is, by virtue of (14), to solve the following optimization problem:

$$
\begin{aligned}
& \underset{\widehat{\mathbf{d}}_{i, j}, z}{\operatorname{maximize}} \quad z, \\
& \text { subject to } \\
& c_{k, \ell}\left(\widehat{\mathbf{d}}_{i, j}\right) \geq z,
\end{aligned}
$$

where $\widehat{\mathbf{d}}_{i, j}$ are inner control points as stated in (10), $c_{k, \ell}$ are given by (14), and $z$ is an auxiliary optimization variable. If $z$ resulting from approximately solving (19) to local optimality is positive then we are guaranteed to have a valid parametrization. Unfortunately, the quality of the parametrization obtained in this fashion needs not to be very high. We can further improve the parametrization by trying to approximate a conformal map. That is, ideally we would like $\mathbf{g}=\mathbf{J}^{T} \mathbf{J}$ to be an identically diagonal matrix (e.g., see [32]).

Let $\lambda_{1}$ and $\lambda_{2}$ be the eigenvalues of the matrix $\mathbf{g}$. Then $\mathbf{g}$ satisfies the ideal condition if and only if $\lambda_{1}=\lambda_{2}$. The identity

$$
\frac{\left(\sqrt{\lambda_{1}}-\sqrt{\lambda_{2}}\right)^{2}}{\sqrt{\lambda_{1} \lambda_{2}}}=\frac{\lambda_{1}+\lambda_{2}}{\sqrt{\lambda_{1} \lambda_{2}}}-2
$$


gives rise to Winslow functional [33]

$$
W=\frac{\lambda_{1}+\lambda_{2}}{\sqrt{\lambda_{1} \lambda_{2}}}=\frac{\operatorname{trace}(\mathbf{g})}{\sqrt{\operatorname{det}(\mathbf{g})}}=\frac{\left\|\mathbf{F}_{u}\right\|^{2}+\left\|\mathbf{F}_{v}\right\|^{2}}{\operatorname{det}\left[\mathbf{F}_{u}, \mathbf{F}_{v}\right]} .
$$

In order to make $\mathbf{g}$ "as identically diagonal as possible" we would like to minimize $W$; however, to ensure the positivity of the determinant we consider the following constrained optimization problem:

$$
\begin{array}{cl}
\underset{\widehat{\mathbf{d}}_{i, j}}{\operatorname{minimize}} & \int_{0}^{1} \int_{0}^{1} W\left(\widehat{\mathbf{d}}_{i, j}\right) \mathrm{d} u \mathrm{~d} v, \\
\text { subject to } & c_{k \ell}\left(\widehat{\mathbf{d}}_{i, j}\right) \geq \delta z_{0} .
\end{array}
$$

In (22), $z_{0}>0$ is computed by approximately solving (19) and $\delta \in[0,1]$ is a fixed relaxation parameter. For further properties of the problem $(22)$, we refer the interested reader to [34].

In our numerical experiments we utilize Matlab's optimization framework for solving optimization problems (19), (22) to approximate stationarity. Also, we set $\delta=0$ in (22).

\section{Isogeometric shape optimization problem}

We consider the problem of finding a shape of a membrane where the first $N$ eigenfrequencies (eigenvalues of the Laplacian) are prescribed. For simplicity we restrict ourselves to domains constituted by one patch. We first discuss different formulations of such a shape optimization problem, and then its sensitivity analysis is carried out.

\subsection{Problem formulation}

The shape of the membrane is fully determined given the parametrization of the boundary. Therefore we let boundary control points be our design variables. One may also require some regularity from the resulting shape; for example, one may be interested in membranes bounded with tangent continuous curves. The latter requirement can be easily fulfilled by enforcing simple constraints on the boundary control points, see [30] Furthermore, "one can not hear the shape of a drum," that is, even if an (admissible) full spectrum is prescribed there could be more than one shape of a membrane that matches it [35]. Therefore, one may expect that there is more than one shape that matches the prescribed few eigenvalues. In order to further restrict the shapes generated by our optimization procedure, we search for a shape, which in addition to satisfying the eigenvalue constraints also has the shortest perimeter. Fig. 3 illustrates the issue. Using this regularization idea, we arrive at the following shape optimization problem:

$$
\begin{array}{rlr}
\underset{\boldsymbol{\rho}_{b}, z}{\operatorname{minimize}} & L\left(\boldsymbol{\rho}_{b}\right), \\
\text { subject to } & \lambda_{k}\left(\boldsymbol{\rho}_{b}\right)=\lambda_{k}^{0}, & \text { if } \lambda_{k}^{0} \text { has multiplicity one, } \\
& \left\{\begin{array}{lr}
\lambda_{k}\left(\boldsymbol{\rho}_{b}\right)+\lambda_{k+1}\left(\boldsymbol{\rho}_{b}\right)=\lambda_{k}^{0}+\lambda_{k+1}^{0} \\
\lambda_{k}\left(\boldsymbol{\rho}_{b}\right) \lambda_{k+1}\left(\boldsymbol{\rho}_{b}\right)=\lambda_{k}^{0} \lambda_{k+1}^{0}
\end{array}\right. & \text { if } \lambda_{k}^{0}=\lambda_{k+1}^{0}, \\
& \operatorname{det}\left[\mathbf{d}_{2}^{j}-\mathbf{d}_{1}^{j}, \mathbf{d}_{2}^{j}-\mathbf{d}_{3}^{j}\right]=0, & j=1, \ldots, 4, \\
\text { where } & \mathbf{K}\left(\boldsymbol{\rho}_{b}\right) \mathbf{u}_{k}=\lambda_{k}\left(\boldsymbol{\rho}_{b}\right) \mathbf{M}\left(\boldsymbol{\rho}_{b}\right) \mathbf{u}_{k}, & \text { for some } \mathbf{u}_{k} \neq \mathbf{0}, k=1, \ldots, N,
\end{array}
$$



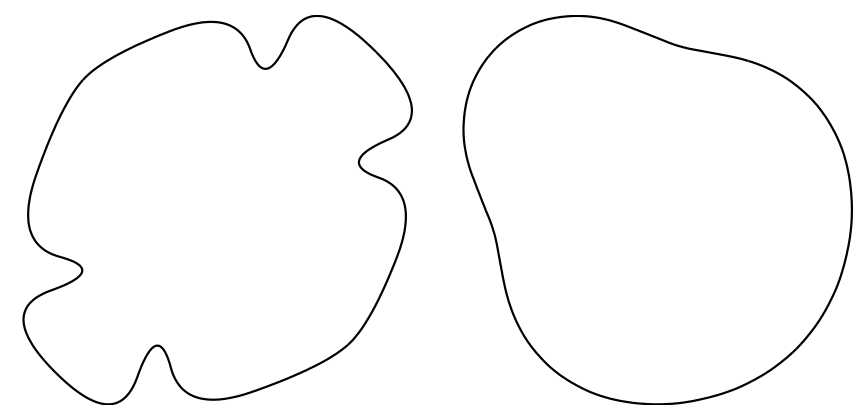

Figure 3: Membranes on the left and on the right have the same five lowest eigenfrequencies, prescribed to be 5.0122, 11.6349, 13.4102, 20.6025 and 23.6877. The shape on the right (a "pear-shaped" region [20]) was obtained by minimizing the perimeter of the domain.

where, $L=L\left(\boldsymbol{\rho}_{b}\right)$ is the perimeter of the shape, $\lambda_{k}^{0}, k=1, \ldots, N$, are prescribed values for the first $N$ eigenvalues, and $\boldsymbol{\rho}_{b}$ are the boundary control points given by (9). The constraints $(23 \mathrm{c})$ are introduced to handle problems with double eigenvalues, c.f. [36]; and the constraints $(23 \mathrm{~d})$ result from enforcing the continuous tangent condition of the boundary at four corners of $[0,1]^{2}$, in which $\mathbf{d}_{1}^{j}, \mathbf{d}_{2}^{j}, \mathbf{d}_{3}^{j}$ is the triple of boundary control points corresponding to the corners.

\subsection{Sensitivity analysis}

In order to utilize gradient-based optimization algorithms for solving (23) numerically, we need to compute derivatives of the constraints entering (23) with respect to changes in boundary control points. If the eigenvalues involved in a constraint have multiplicity one, then one may derive, using (7) and (8), that

$$
\frac{\partial \lambda_{k}}{\partial \widehat{\rho}}=\mathbf{u}_{k}^{T}\left(\frac{\partial \mathbf{K}}{\partial \widehat{\rho}}-\lambda_{k} \frac{\partial \mathbf{M}}{\partial \widehat{\rho}}\right) \mathbf{u},
$$

where $\hat{\rho}$ is an arbitrary component of the vector $\widehat{\boldsymbol{\rho}}$, and $\lambda_{k}$ and $\mathbf{u}_{k}$ are an eigenvalue and an eigenvector of (7), (8). However, if the multiplicity of an eigenvalue $\lambda_{k}$ may change during the optimization process, then the individual eigenvalues may not be differentiable functions of the design variables any longer. Nevertheless, it may still be possible to differentiate certain functions of the eigenvalues. It can be shown [36] that the sensitivities of the functions $\lambda_{k}+\lambda_{k+1}$ and $\lambda_{k} \lambda_{k+1}$ are given as

$$
\begin{aligned}
\frac{\partial\left(\lambda_{k}+\lambda_{k+1}\right)}{\partial \widehat{\rho}} & =\mathbf{u}_{k}^{T}\left(\frac{\partial \mathbf{K}}{\partial \widehat{\rho}}-\lambda_{k} \frac{\partial \mathbf{M}}{\partial \widehat{\rho}}\right) \mathbf{u}_{k}+\mathbf{u}_{k+1}^{T}\left(\frac{\partial \mathbf{K}}{\partial \widehat{\rho}}-\lambda_{k+1} \frac{\partial \mathbf{M}}{\partial \widehat{\rho}}\right) \mathbf{u}_{k+1}, \\
\frac{\partial\left(\lambda_{k} \lambda_{k+1}\right)}{\partial \widehat{\rho}} & =\lambda_{k+1} \mathbf{u}_{k}^{T}\left(\frac{\partial \mathbf{K}}{\partial \widehat{\rho}}-\lambda_{k} \frac{\partial \mathbf{M}}{\partial \widehat{\rho}}\right) \mathbf{u}_{k}+\lambda_{k} \mathbf{u}_{k+1}^{T}\left(\frac{\partial \mathbf{K}}{\partial \widehat{\rho}}-\lambda_{k+1} \frac{\partial \mathbf{M}}{\partial \widehat{\rho}}\right) \mathbf{u}_{k+1} .
\end{aligned}
$$

From (9) and (15), it follows that if the partial derivative of a function $f$ with respect to $\widehat{\rho}$ has been calculated as above, its sensitivities with respect to the design variables $\boldsymbol{\rho}_{b}$ are given by

$$
\frac{\partial f}{\partial \boldsymbol{\rho}_{b}}=\frac{\partial f}{\partial \widehat{\boldsymbol{\rho}}_{b}} \underset{9}{\mathbf{T}}=\frac{\partial f}{\partial \widehat{\boldsymbol{\rho}}} \mathbf{G} \mathbf{T} .
$$


Other methods of dealing with multiple eigenvalues are discussed in [37-40].

\section{Numerical examples}

In this section, we discuss our strategy for solving (23), and then illustrate its practical performance by solving a few shape optimization problems for vibrating membranes.

\subsection{Solution strategy}

We use the following approach for solving (23):

(1) Preparing a good initial shape: We want to find a good initial shape in a "cheap" way and use it for more expensive computational work. To this end, we use a small number of control points to approximately solve (23). The constraint tolerance $T_{\text {con }}$ is set to be around $10^{-4}-10^{-6}$. After a suitable shape is found, we disregard the perimeter, look only at the eigenvalues, and use a very small constraint tolerance $T_{\text {con }}$ around $10^{-14}-10^{-16}$. After a feasible initial shape is found, we refine the computational grid by inserting more knots into the knot vectors.

(2) Improving the initial parametrization: If the quasi-conformal deformation method is chosen, the control net of the initial shape will be used as the reference configuration during the optimization iterations. Since the mesh regularity affects the accuracy of the numerical solution $[8,41]$, it is necessary to improve the parametrization of the reference configuration as discussed in Section 3.4. Fig. 10 demonstrates the necessity.

(3) Performing optimization: We determine the knot vectors $\widetilde{\boldsymbol{\Xi}}_{u}$ and $\widetilde{\boldsymbol{\Xi}}_{v}$ by halving the knot intervals of the knot vectors $\widehat{\boldsymbol{\Xi}}_{u}$ and $\widehat{\boldsymbol{\Xi}}_{v}$ several times such that further halving does not change the calculated eigenvalues by more than a given tolerance $T_{c v}$. The optimization problem is then solved numerically with the obtained knot vectors and initial shape.

Throughout the present work, numerical integration is done by using standard Gaussian quadratures. The homogeneus Dirichlet boundary conditions are enforced by setting the corresponding boundary state variables to zero [6].

All the solutions presented in this section have been obtained with gradient based non-linear programming solver fmincon, which is a part of the optimization toolbox of Matlab, version 7.5.0 (R2007b) [42].

\subsection{Pear-shaped region}

We start with an example of a membrane with the lowest five eigenvalues prescribed to be $5.0122,11.6349,13.4102,20.6025$, and 23.6877. This numerical example has been considered in Hutchinson et al. [20]. The initial shape shown in Fig. 4 has been obtained by "rounding" the rectangle $[-3 / \pi, 3 / \pi] \times[-1,1]$. This choice of the initial shape ensures that its first five eigenvalues, which are 5.2202, 12.7756, 13.4938, 21.3752, 26.0602, are close to the target ones and have multiplicity one. The latter allows us to avoid the issues with double eigenvalue discussed in Section 4. To compute a good initial shape as described in Step (1), Section 5.1, we use the same knot vectors for the geometry, parametrization and the solution space

$$
\boldsymbol{\Xi}_{s}=\boldsymbol{\Xi}_{t}=\widehat{\boldsymbol{\Xi}}_{s}=\widehat{\boldsymbol{\Xi}}_{t}=\widetilde{\boldsymbol{\Xi}}_{s}=\widetilde{\boldsymbol{\Xi}}_{t}=\{0,0,0,0.25,0.5,0.75,1,1,1\} .
$$




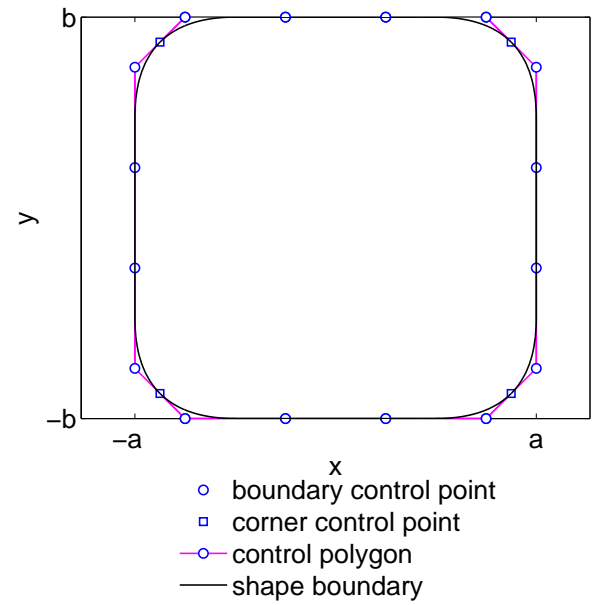

Figure 4: Initial shape

The spring model is used for extending the parametrization from boundary to the interior of the domain. The B-splines for representing the parametrization and solution space are quadratic. This setting yields a $6 \times 6$ control net, 16 elements and 16 degrees of freedom (dof). The optimization process with $T_{\text {con }}=10^{-7}$ finishes after 290 iterations. The final shape is shown in Fig. 5; its overall features are fairly similar to that of [20].

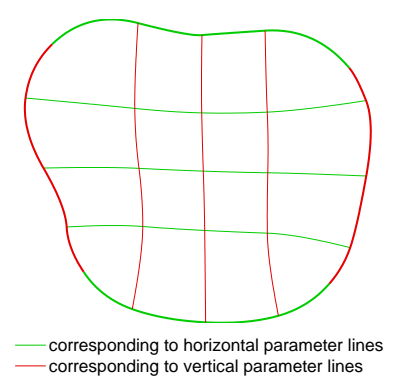

(a)

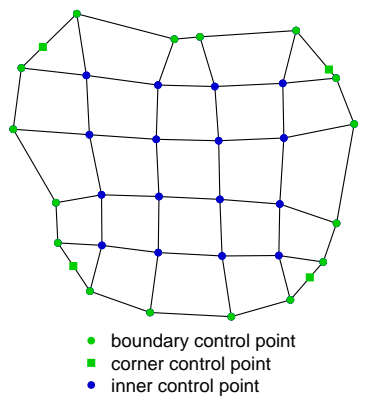

(b)

Figure 5: (a): Optimized shape and (b): the corresponding control net of a pear-shape region.

The solution shown in Fig. 5 is further refined and is used a new initial design. The refinement is done by halving all knot intervals of all knot vectors. The new setting has $10 \times 10$ control points, 64 elements and 64 degrees of freedom. The optimal shape is depicted in Fig. 6 (a)-(b); this shape is closer to that of [20].

The optimized shape in Fig. 6 (a) is nearly symmetric about the straight line connecting its two opposite corner control points, see Fig. 6 (b). To investigate the robustness of the isogeometric shape optimization in preserving a line symmetry, we resolve the shape 
optimization problem this time explicitly imposing the shape to be symmetric about the line $x+y=0$. This is done by imposing the boundary control points of the shape to be symmetric about the line. Repeating the same procedure as with the last design (with the same initial shape in Fig. 5), but with symmetry constraints, we obtain an optimized shape Fig. 6 (c)-(d). Both designs, with and without explicit symmetry constraints, agree very well both qualitatively (Fig. 6) and quantitatively (Tab. 1).

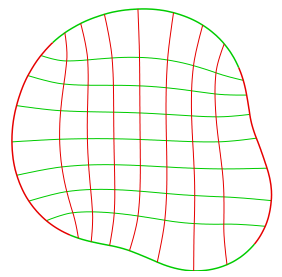

(a)

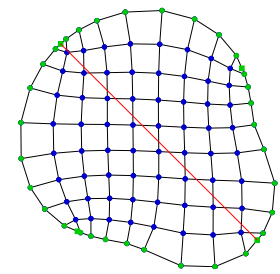

(b)

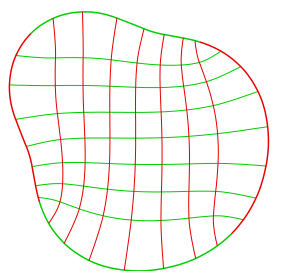

(c)

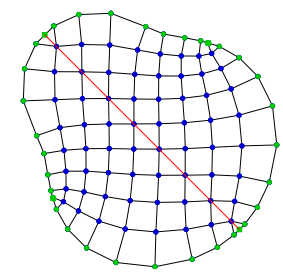

(d)

Figure 6: (a), (c): optimized shapes and (b), (d): corresponding control nets for a pear-shaped region example. (a), (b): design computed without explicit symmetry constraints; (c), (d): design computed with explicit symmetry constraint.

\begin{tabular}{lccccccc}
\hline Design & $\widetilde{p}$ & \# var. & dof & \# iter. & relative error & perimeter & area \\
\hline \hline Initial (Fig. 6) & 2 & - & 64 & - & 0.019 & 7.1592 & 3.8208 \\
Without sym. & 2 & 72 & 64 & 340 & $6.1 \times 10^{-8}$ & 7.0380 & 3.7686 \\
With sym. & 2 & 72 & 64 & 340 & $2.3 \times 10^{-9}$ & 7.0378 & 3.7686 \\
\hline
\end{tabular}

Table 1: Quantitative comparison of the optimized pear-shaped membranes without and with explicit enforcing of symmetry. \# var.: Number of design variables; \# iter.: number of optimization iterations needed to achieve convergence.

\subsection{Harmonic drums}

Another interesting and more challenging example of shape optimization is the problem of finding a shape of a harmonic drum [20]. That is, we are looking for a membrane such that when "played" its lowest eigenfrequencies correspond to the notes $\mathrm{C}_{1}, \mathrm{G}_{1}$, and $\mathrm{C}_{2}$ on the Pythagorean Scale, c.f. [27, Chapter 4]. Since repeating the second note should not change the sound, we look for a membrane with the first four eigenfrequencies constituting a ratio $2: 3: 3: 4$ (c.f. [20]).

\subsubsection{One patch design}

In this example, we start with an initial shape similar to that of the pear-shaped region case, see Fig. 5 . The only difference is that the rectangle $[-3 / \pi, 3 / \pi] \times[-1,1]$ is replaced by the square $[-1,1]^{2}$. The first four eigenvalues of the shape are $4.9844,12.5403,12.6183$ and 20.537. Choosing the second initial eigenvalue as a reference frequency, we calculate the prescribed eigenvalues as 5.5735, 12.5403, 12.5403, and 22.2939. Bi-quadratic Bspline parametrizations with respect to the knot vectors (28) are used for the initial design. The extension of a parametrization from the domain boundary to the interior 
is done by the quasi-conformal deformation method. The first reference configuration is depicted in Fig. 7 (a), where we have introduced a slight asymmetry. In our experience, this helps to accelerate the convergence of the optimization algorithm.

The optimized shape is shown in Fig. 7. For the resulting control net, we examine

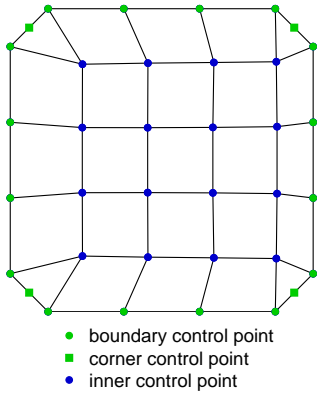

(a)

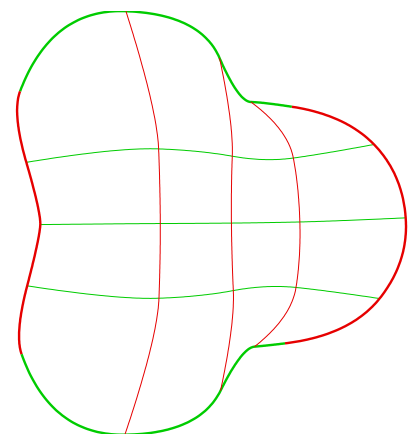

(b)

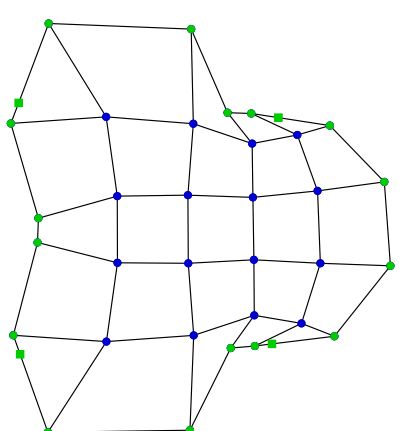

(c)

Figure 7: Optimized design of a harmonic drum. (a): Initial reference control net. (b): Optimized shape and (c): the corresponding control net.

the validity of the corresponding parametrization. We compute the coefficients $c_{k, \ell}$ in the expansion of the determinant of its Jacobian, given by (14). Only three out of 28561 control points are negative, see Fig. 8 (a). Furthermore, after refining the grid, the new coefficients $c_{k, \ell}$ are all positive, see Fig. 8 (b).

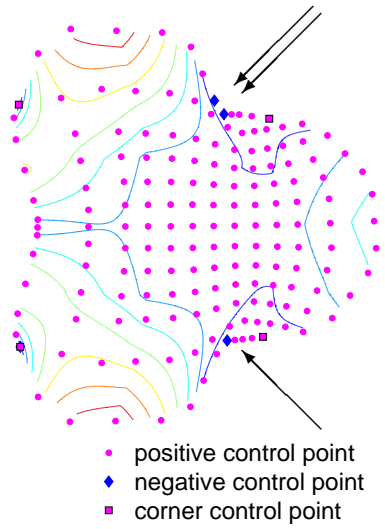

(a)
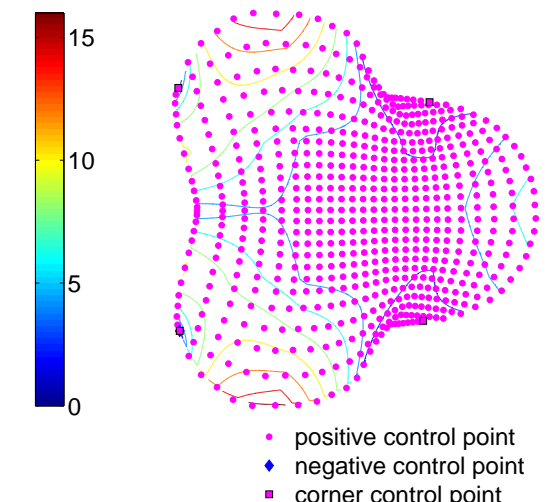

(b)

Figure 8: Illustration of the validation of the B-spline parametrization shown in Fig. 7. (a): The expansion coefficients and contour lines of the Jacobian determinant corresponding to the original control net; (b): the same for the refined control net.

After neglecting the perimeter and only optimizing eigenvalues, a very similar shape is obtained. Refining the grid and starting the optimization algorithm from the last 
obtained shape we get a drum shown in Fig. 9. When we use the control net in Fig. 9 as

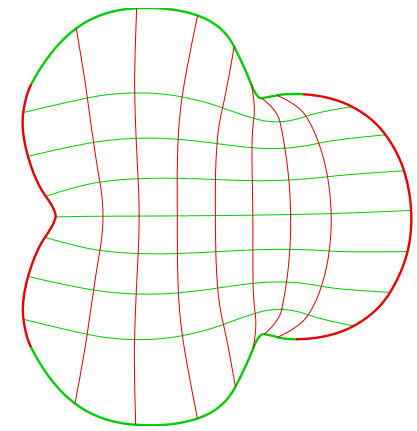

(a)

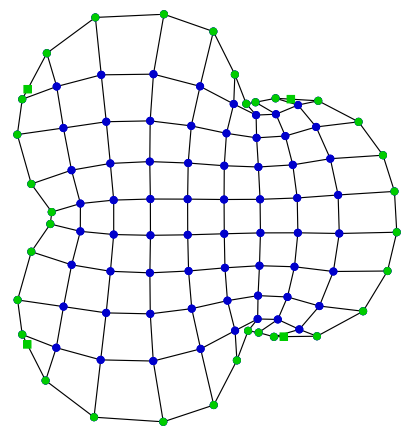

(b)

Figure 9: (a): Optimized shape and (b): control net for a further refinement of a harmonic drum shape optimization problem.

a reference net, we find that an invalid parametrization appeared after a few optimization iterations. Therefore, it is crucial to improve the resulting parametrization utilizing either the optimization problem (19) or (22). This leads to the different configurations shown in Fig. 10. The solution space is chosen by halving the knot intervals three times. Finally, resolving the optimization problem results in a shape shown in Fig. 11. The first four eigenmodes of the shape are depicted in Fig. 12. See Tab. 2 and 3 for more quantitative data on the design. Corners in the shapes in Fig. 11 (dof $=4096$ ) are sharper than those in the one shown in Fig. 7 (dof $=16)$, meaning that the last refinement step was necessary.

\subsubsection{Three patch design}

The resulting shapes in Fig. 11 enjoy a nearly $120^{\circ}$ symmetry. To further explore the problem, we impose the exact $120^{\circ}$ symmetry by modeling the shape with three identical patches as shown in Fig. 13. The exact symmetry implies that $\lambda_{2}=\lambda_{3}$ at all times, and therefore this double eigenvalue remains differentiable with respect to the design parameters. For the first design problem, we use bicubic B-splines with knot vectors

$$
\boldsymbol{\Xi}_{s}=\boldsymbol{\Xi}_{t}=\widehat{\boldsymbol{\Xi}}_{s}=\widehat{\boldsymbol{\Xi}}_{t}=\widetilde{\boldsymbol{\Xi}}_{s}=\widetilde{\boldsymbol{\Xi}}_{t}=\{0,0,0,0,0.25,0.5,0.75,1,1,1,1\} .
$$

The spring model is used to generate the inner control points. The initial shape of the design is shown in Fig. 13. We follow the scheme outlined in Section 5.1. After performing the first step, we obtain a shape shown in Fig. 14. We continue by using the resulting shape as a new initial shape and halving the knot intervals three times. After performing the optimization on a refined geometry, we obtain a drum shown in Fig. 15. The resulting shape is symmetric about the line connecting the two opposite corner control points, see Fig. 15 (b), even though we did not explicitly impose the symmetry requirement. One also observes that the resulting parametrization is valid, see Fig. 15 (c).

We again investigate the effects on the results of the two optimization strategies, that is, just matching the eigenvalues (disregarding the perimeter) vs. minimizing the perimeter in addition to matching the eigenvalues (problem (23)). In this numerical 

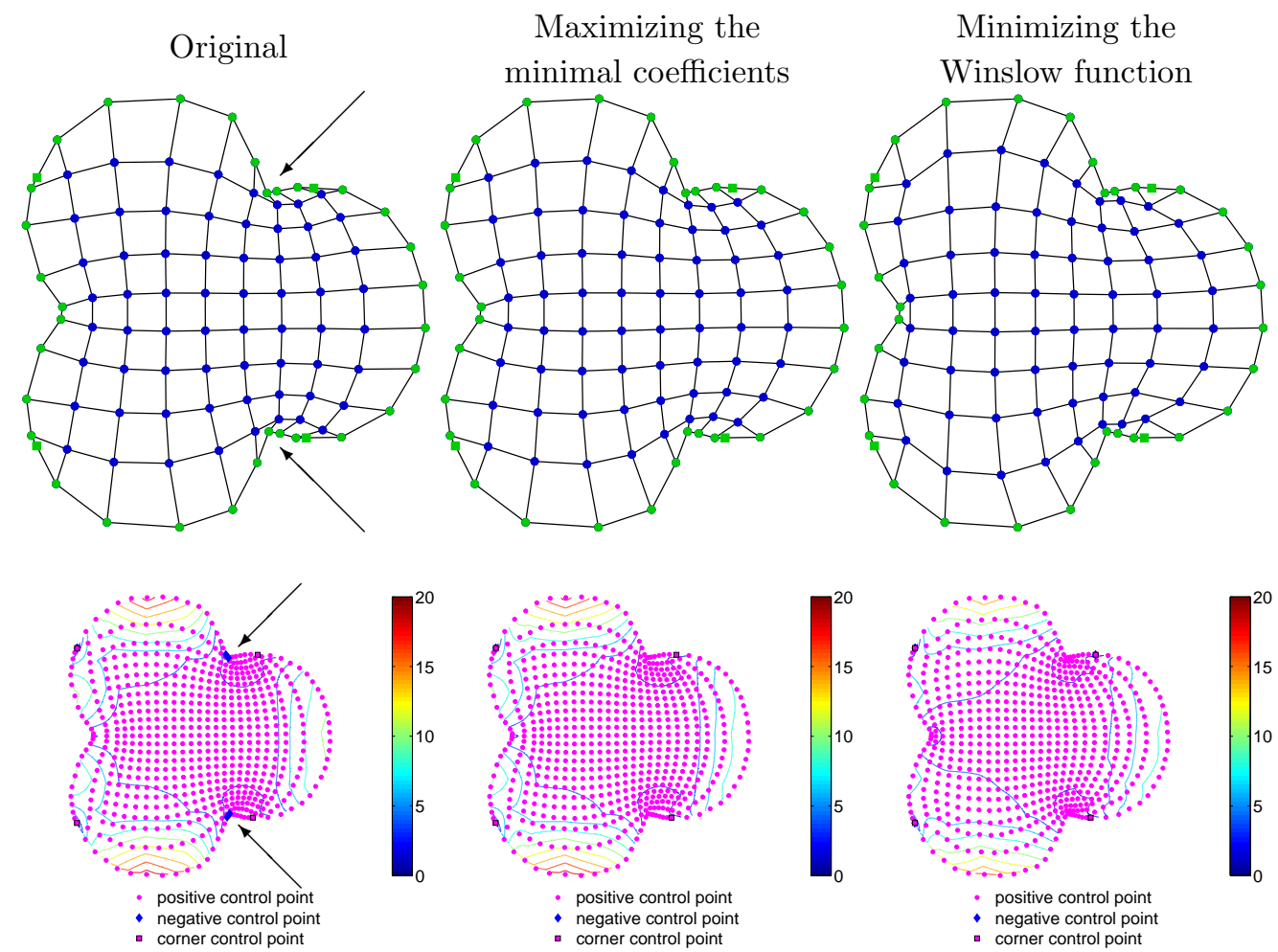

Figure 10: Various ways of improving the B-spline parametrization. Upper row: Control nets; lower row: corresponding expansion coefficients and contour lines of the Jacobian determinant.

experiment we use the knot vectors which are given by (29), the B-splines of degree three, resulting in dof $=91$. The constraint tolerances are set to $T_{\text {con }}=10^{-7}$. We start the optimization algorithm from two initial points, and obtain final shapes shown in Fig. 16. One may observe that the numerical solutions to the optimization problem where only eigenvalue matching is optimized are extremely sensitive with respect to the choice of the initial shapes, whereas this is not the case with the optimization problem (23).

We now measure the errors in eigenvalues achieved by the different designs. Let $\lambda_{i}$, $i=1,2,3,4$, be resulting eigenvalues of an optimized shape. We use $\lambda_{2}=\lambda_{2}^{0}$ as the normalization factor to obtain the values $\lambda_{i}^{0}, i=1,2,3,4$. Then we compute the relative error corresponding to a given design as

$$
\text { relative error }=\max _{i=1,2,3,4} \frac{\left|\lambda_{i}-\lambda_{i}^{0}\right|}{\lambda_{i}^{0}} .
$$

The relative errors and the normalized first four frequencies of the designs presented above are summarized in Tab. 2 and 3, respectively. Tab. 2 shows that the designs computed using a three-patch approach achieve better accuracy with respect to the eigenvalues. This matches well the theoretical prediction that we have exact double eigenvalues due to the symmetry. It is also observed that the drum in Fig. 15 has gross features similar 


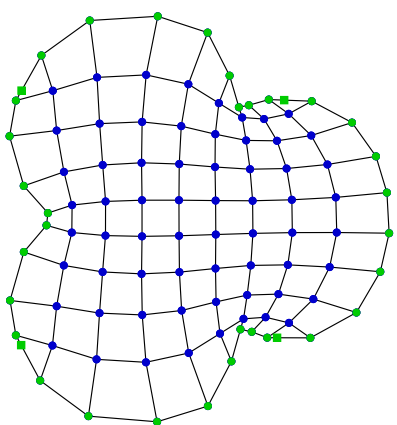

(a)

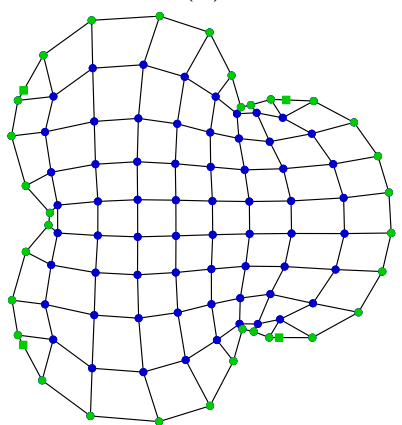

(c)

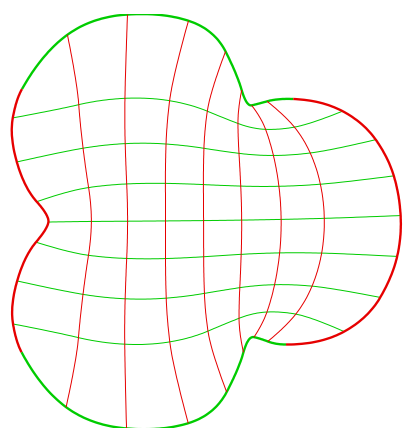

(b)

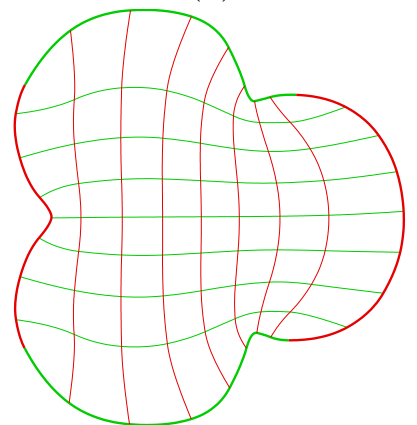

(d)

Figure 11: (a), (c): The final optimized shapes and (b), (d): the corresponding control nets of a harmonic drum. (a) \& (b): results corresponding to the reference control net in Fig. 10 (b); (c) \& (d): results corresponding to the reference control net in Fig. 10 (c).

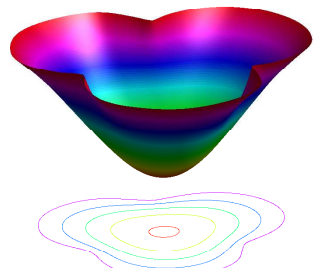

$f_{1}=2.00007$

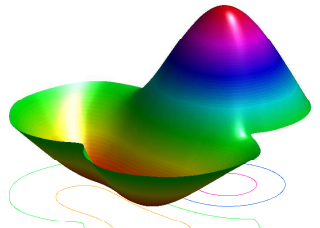

$f_{2}=3.00000$

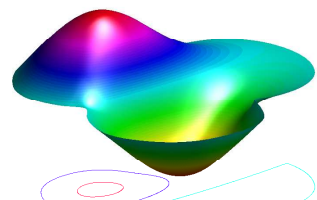

$f_{3}=3.00023$

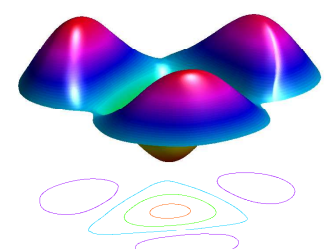

$f_{4}=4.00015$

Figure 12: The first four eigenmodes and normalized frequencies of the optimized shape in Fig. 11 (d).

to those of [20] but with far more satisfactory frequencies $\left(120^{\circ}\right.$-symmetry is imposed in the both cases).

\subsection{CEG drums}

Finally, we design a different kind of a harmonic drum. Namely, we seek a shape capable of "playing" the musical triad C-E-G (c.f. [27]), corresponding to the ratio 4:5:5:6 of 


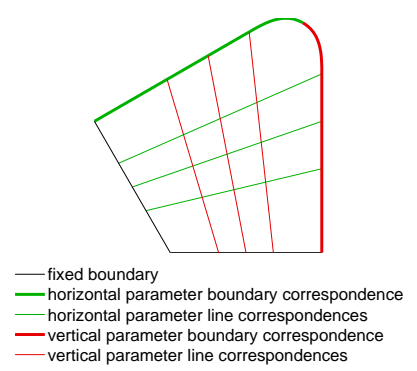

(a)

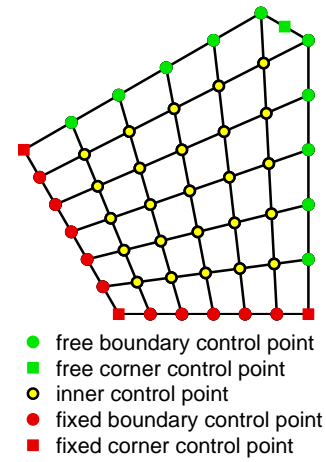

(b)

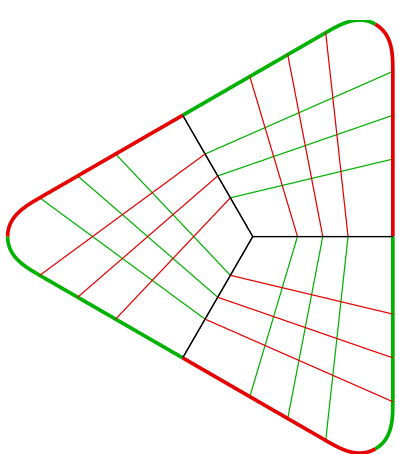

(c)

Figure 13: Initial shape for the three-patch design of the harmonic drum. (a): Generating patch and (b): the corresponding control net. (c): Entire domain consisting of the generating patch repeated three times.

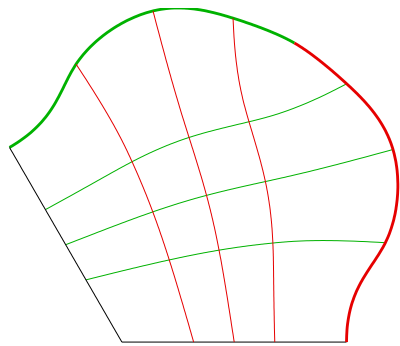

(a)

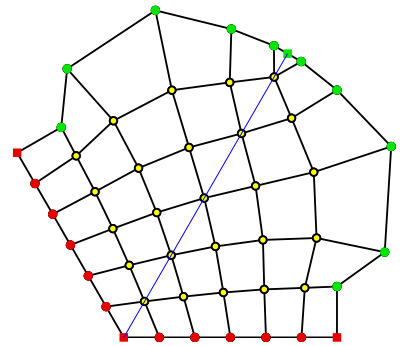

(b)

Figure 14: (a): Optimized shape and (b): the corresponding control net of a three-patch design of a harmonic drum.

\begin{tabular}{lcccccc}
\hline Design & \# refinements & $\widetilde{p}$ & \# var. & dof & \# iter. & relative error \\
\hline \hline Fig. 4 (initial) & 3 & 2 & - & 4096 & - & 0.1148 \\
Fig. 11 (b) (1 patch) & 3 & 2 & 72 & 4096 & 17 & $1.6 \times 10^{-4}$ \\
Fig. 11 (d) (1 patch) & 3 & 2 & 72 & 4096 & 16 & $1.6 \times 10^{-4}$ \\
Fig. 13 (initial) & 3 & 3 & - & 3367 & - & 0.0380 \\
Fig. 15 (3 patches) & 3 & 3 & 22 & 3367 & 31 & $1.5 \times 10^{-13}$ \\
Harmonic drum of [20] & - & - & 14 & 60 & - & $0.7 \times 10^{-4}$ \\
\hline
\end{tabular}

Table 2: Quantitative data for various designs of the harmonic drum. \# refinements: Number of halving the knot intervals; $\widetilde{p}$ : degrees of the B-splines used for forming the solution space; \# var.: number of design variables; \# iter.: number of optimization iterations needed to achieve convergence.

the first four natural frequencies. We start by using the same initial shape and knot vectors as in the last example. The B-splines for geometry and solution space are quadratic. Following the optimization scheme outlined in Section 5.1 we obtain the shapes shown 


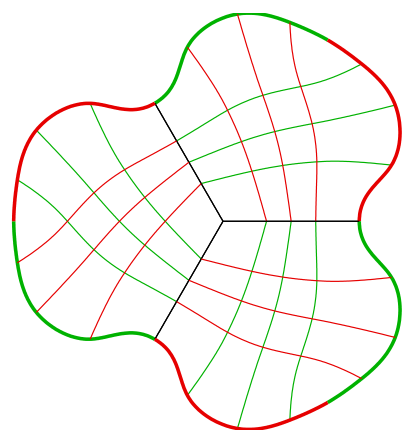

(a)

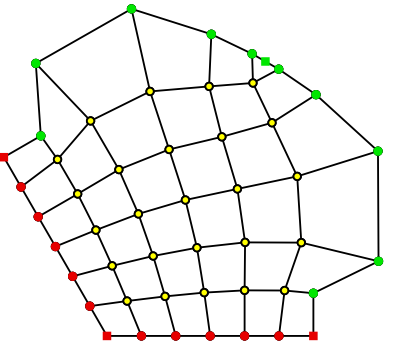

(b)

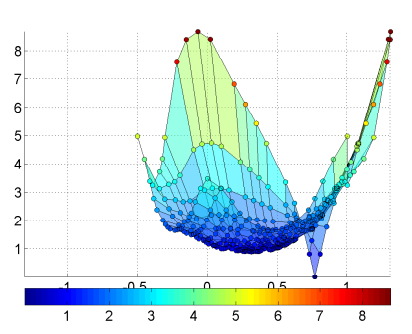

(c)

Figure 15: Refined and optimized shape of a three-patch design of a harmonic drum. (a): Optimal generating patch and (b): the corresponding control net. (c): Coefficients in the expansion of the determinant of the Jacobian. The non-positive (zero) coefficient is the corner control point where the smoothness constraints (23d) force a singularity.

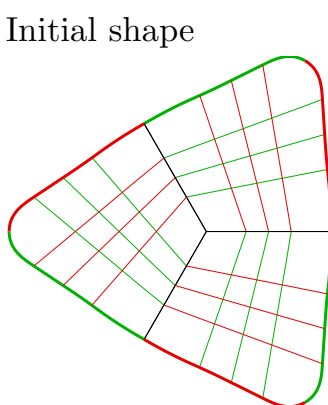

perimeter: 8.526 area: 4.5835

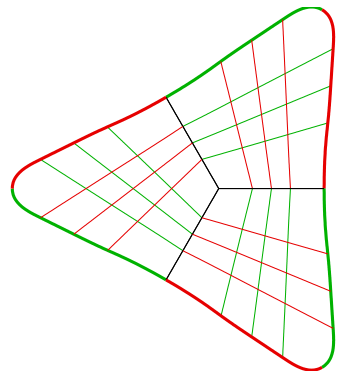

perimeter: 10.419 area: 5.6535
Optimizing eigenvalues

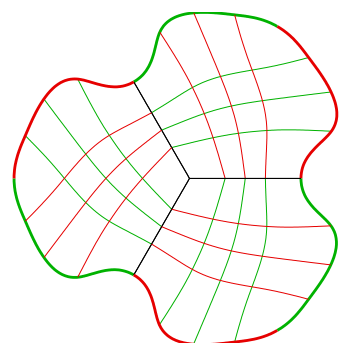

perimeter: 9.9435 area: 6.0869

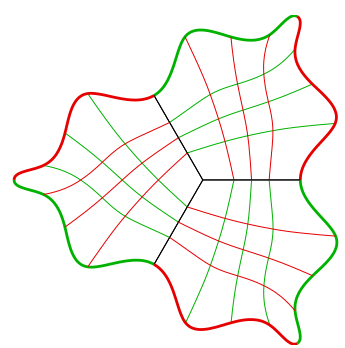

perimeter: 11.7512 area: 6.3733
Minimizing the perimeter

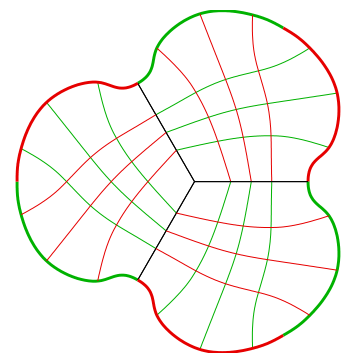

perimeter: 9.7767 area: 6.0725

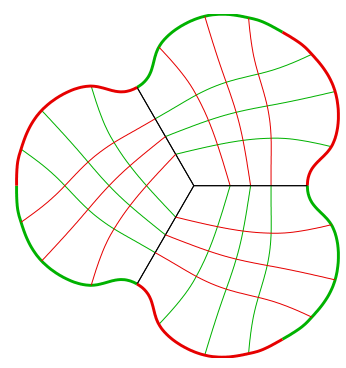

perimeter: 9.7845 area: 6.088

Figure 16: Comparison between different optimization strategies with different initial shapes.

in Fig. 17. In this example refined the geometry by halving the knot intervals two times, which leads to a problem with 817 degrees of freedom. The first four normalized fre- 


\begin{tabular}{lcccccc}
\hline Design & $f_{1}$ & $f_{2}$ & $f_{3}$ & $f_{4}$ & perimeter & area \\
\hline \hline Fig. 4 (initial) & 1.89426 & 3.00000 & 3.00024 & 3.82078 & 7.2465 & 3.8333 \\
Fig. 11b (1 patch) & 2.00008 & 3.00000 & 3.00024 & 4.00016 & 7.6232 & 4.0255 \\
Fig. 11d (1 patch) & 2.00007 & 3.00000 & 3.00023 & 4.00015 & 7.6232 & 4.0276 \\
Fig. 13 (initial) & 1.96351 & 3.00000 & 3.00000 & 3.93126 & 9.3460 & 5.0617 \\
Fig. 15 (3 patches) & 2.00000 & 3.00000 & 3.00000 & 4.00000 & 9.8592 & 6.0686 \\
Harmonic drum of [20] & 2.00011 & 3.00000 & 3.00000 & 3.99972 & - & - \\
\hline
\end{tabular}

Table 3: The first four normalized eigenfrequencies, the length of the perimeter, and the area of the different computed optimal shapes for the harmonic drum example.

quencies of the found optimized shape are 3.99999, 5.00000, 5.00000, and 5.99999, as required.

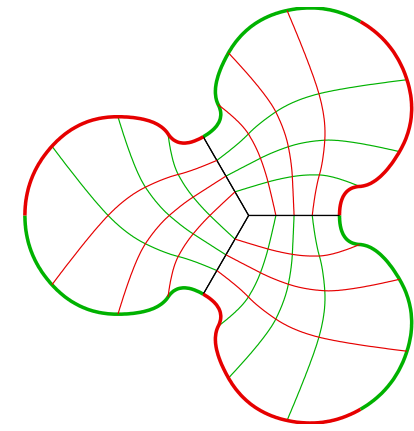

(a)

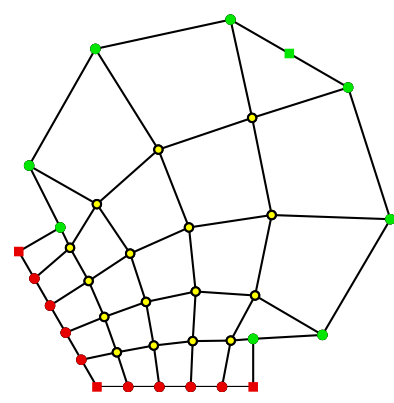

(b)

Figure 17: (a): Optimized shape of a CEG drum; (b): control net corresponding to the generating patch.

\section{Conclusions}

We have applied isogeometric analysis towards shape optimization problems of vibrating membranes, where we allow the shape of the domain to experience very large deformations. We have used three different B-spline parametrizations for the boundary and the interior of the domains, as well as for the approximate solution space for the governing boundary value problem. This allows us to independently vary the number of control points for the shape parametrization and the approximation properties of the discretization of the governing equations.

We have carried out numerical studies of several techniques for extending the domain parametrization from the boundary to the interior and improving the quality of the domain parametrization. As a result, we have presented solutions to several eigenvalue optimization problems for the Laplace equation. Due to the properties of B-splines, some nice shapes have been obtained with a relatively small number of design variables. The problems have been solved numerically in a number of different ways (for example, 
without and with explicit constraints ensuring certain symmetries) yet they yielded the same optimal solutions, thus supporting the robustness of our approach.

\section{Acknowledgements}

The authors thank the members of the TopOpt group (www.topopt.dtu.dk) for fruitful discussions related to this paper. The financial support from the Danish Center for Scientific Computing (DCSC) is gratefully acknowledged.

\section{References}

[1] M.C. Delfour and J.P. Zolésio. Shapes and geometries. Analysis, differential calculus, and optimization, volume 4 of Advances in Design and Control. Society for Industrial and Applied Mathematics (SIAM), Philadelphia, PA, 2001.

[2] B. Mohammadi and O. Pironneau. Applied Shape Optimization for Fluids. Oxford University Press, 2001.

[3] Grégoire Allaire. Shape optimization by the homogenization method, volume 146 of Applied Mathematical Sciences. Springer-Verlag, New York, 2002.

[4] M.P. Bendsøe and O. Sigmund. Topology optimization. Theory, methods and applications. SpringerVerlag, Berlin, 2003.

[5] J.A. Sethian. Level Set Methods and Fast Marching Methods : Evolving Interfaces in Computational Geometry, Fluid Mechanics, Computer Vision, and Materials Science. Cambridge University Press, 1999.

[6] T.J.R. Hughes, J.A. Cottrell, and Y. Bazilevs. Isogeometric analysis: CAD, finite elements, NURBS, exact geometry and mesh refinement. Comput. Methods Appl. Mech. Engrg., 194(39-41):4135-4195, 2005.

[7] J.A. Cottrell, A. Reali, Y. Bazilevs, and T.J.R. Hughes. Isogeometric analysis of structural vibrations. Comput. Methods Appl. Mech. Engrg., 195(41-43):5257-5296, 2006.

[8] Y. Bazilevs, L. Beirão da Veiga, J.A. Cottrell, T.J.R. Hughes, and G. Sangalli. Isogeometric analysis: approximation, stability and error estimates for $h$-refined meshes. Math. Models Methods Appl. Sci., 16(7):1031-1090, 2006.

[9] T.J.R. Hughes, A. Reali, and G. Sangalli. Duality and unified analysis of discrete approximations in structural dynamics and wave propagation: comparison of $p$-method finite elements with $k$-method NURBS. Comput. Methods Appl. Mech. Engrg., 197(49-50):4104-4124, 2008.

[10] W.A. Wall, M.A. Frenzel, and C. Cyron. Isogeometric structural shape optimization. Comput. Methods Appl. Mech. Engrg., 197(33-40):2976-2988, 2008.

[11] Seonho Cho and Seung-Hyun Ha. Isogeometric shape design optimization: exact geometry and enhanced sensitivity. Struct. Multidiscip. Optim., 38(1):53-70, 2009.

[12] A.P. Nagy, M.M. Abdalla, and Z. Gurdal. Isogeometric sizing and shape optimisation of beam structures. Comput. Methods Appl. Mech. Engrg., 199(17-20):1216-1230, 2010.

[13] Xiaoping Qian. Full analytical sensitivities in NURBS based isogeometric shape optimization. Computer Methods in Applied Mechanics and Engineering, In Press, Corrected Proof:-, 2010.

[14] Yuri Bazilevs J.A. Cottrell, T.J.R. Hughes. Isogeometric Analysis: Toward Integration of CAD and FEA. J. Wiley., West Sussex, 2009.

[15] Antoine Henrot. Minimization problems for eigenvalues of the Laplacian. J. Evol. Equ., 3(3):443461, 2003.

[16] Zakaria Belhachmi, Dorin Bucur, Giuseppe Buttazzo, and Jean-Marc Sac-Epée. Shape optimization problems for eigenvalues of elliptic operators. ZAMM Z. Angew. Math. Mech., 86(3):171-184, 2006.

[17] N.L. Pedersen. Optimization of holes in plates for control of eigenfrequencies. Struct. Multidiscip. Optim., 28(1):1-10, AUG 2004.

[18] N.L. Pedersen. On simultaneous shape and orientational design for eigenfrequency optimization. Struct. Multidiscip. Optim., 33(4-5):387-399, APR 2007.

[19] Zhixue Wu. Optimal hole shape for minimum stress concentration using parameterized geometry models. Struct. Multidiscip. Optim., 37(6):625-634, FEB 2009.

[20] J.W. Hutchinson and F.I. Niordson. Designing vibrating membranes. In Continuum mechanics and related problems of analysis (Russian), pages 581-590. Izdat. "Nauka", Moscow, 1972. 
[21] S.A. Wolf and J.B. Keller. Range of the first two eigenvalues of the laplacian. Proceedings: Mathematical and Physical Sciences, 447(1930):397-412, 1994.

[22] Dorin Bucur. Do optimal shapes exist? Milan J. Math., 75:379-398, 2007.

[23] Couro Kane and Marc Schoenauer. A drum shape optimisation by genetic algorithms. Complexity International, 2, 1995.

[24] Yunliang Ding. Shape optimization of structures: a literature survey. Computers E Structures, 24(6):985 - 1004, 1986.

[25] V. Braibant and C. Fleury. Shape optimal design using B-splines. Comput. Methods Appl. Mech. Engrg., 44(3):247 - 267, 1984.

[26] L.E. Kinsler, A.R. Frey, A.B. Coppens, and J.V. Sanders. Fundamentals of Acoustics. John Wiley \& Sons, Singapore, 1982.

[27] D.R. Lapp. The Physics of Music and Musical Instruments. Wright Center for Innovative Science Education, Tufts University, Medford, Massachusetts, 2003.

[28] R.A. Adams. Sobolev spaces. Pure and Applied Mathematics, Vol. 65. Academic Press, New York-London, 1975.

[29] O.C. Zienkiewicz and R.L. Taylor. The finite element method. Vol. 1. The basis. ButterworthHeinemann, Oxford, fifth edition, 2000.

[30] Les Piegl and Wayne Tiller. The NURBS book. Monographs in Visual Communication. Berlin: Springer-Verlag. xiv, 1995

[31] C. de Boor and G.J. Fix. Spline approximation by quasiinterpolants. J. Approximation Theory, 8:19-45, 1973 .

[32] L.V. Ahlfors. Complex analysis. An introduction to the theory of analytic functions of one complex variable, International Series in Pure and Applied Mathematics. McGraw-Hill Book Co., New York, third edition, 1978.

[33] P. Knupp and S. Steinberg. Fundamentals of Grid Generation. CRC Press, Boca Ranton, 1993.

[34] J. Gravesen, A. Evgrafov, and N.D. Manh. Parametrisation in 2-dimensional isogeometric analysis. In preparation.

[35] C. Gordon, D.L. Webb, and S. Wolpert. One cannot hear the shape of a drum. Bull. Amer. Math. Soc. (N.S.), 27(1):134-138, 1992.

[36] J. Gravesen, A. Evgrafov, and N.D. Manh. On the sensitivities of multiple eigenvalues. In preparation.

[37] A.P. Seyranian, E. Lund, and N. Olhoff. Multiple eigenvalues in structural optimization problems. Structural Optimization, 8:207-227, 1994

[38] N.L. Pedersen and A.K. Nielsen. Optimization of practical trusses with constraints on eigenfrequencies, displacements, stresses, and buckling. Struct. Multidiscip. Optim., 25(5-6):436-445, DEC 2003.

[39] J.S. Jensen and N.L. Pedersen. On maximal eigenfrequency separation in two-material structures: the 1D and 2D scalar cases. J. of Sound and Vibration, 289(4-5):967-986, FEB 72006.

[40] Jianbin Du and Niels Olhoff. Topological design of freely vibrating continuum structures for maximum values of simple and multiple eigenfrequencies and frequency gaps. Struct. Multidiscip. Optim. 34(2):91-110, AUG 2007.

[41] A. Buffa, G. Sangalli, and R. Vazquez. Isogeometric analysis in electromagnetics: B-splines approximation. Comput. Methods Appl. Mech. Engrg., 199(17-20):1143-1152, 2010.

[42] http://www.mathworks.com 